\title{
The Effect of Ligands on Noble Metal Nanoparticles as Drug Delivery Systems to the Brain
}

\author{
Shailee Shroff ${ }^{1}$, Chad D. Curtis ${ }^{2 \#}$ \\ ${ }^{1}$ Windermere Preparatory School, Windermere, FL, USA \\ ${ }^{2}$ University of Washington, Seattle, WA 98195 \\ \#Advisor
}

\section{ABSTRACT}

Brain diseases affects 1 and 6 people and generates over a trillion dollars in treatment. Noble metal nanoparticles have been used to address these diseases in the brain, however very few of these formulations have made it through clinical trials. This review will be discussing the role of noble metal nanoparticles as drug delivery systems specifically to the brain. A common problem many researchers and clinical physicians are facing problems because they are unable to access the brain without highly invasive surgery. Nanoparticles allow access to the brain without invasive surgery. Noble metal nanoparticles (NMNPs) are of particular interest because of their inherent characteristics which are amplified or reduced by ligands. The various ligands available change the method of transportation for a NMNPs traveling through the blood barrier. We will examine various ligands and their benefits and potential drawbacks. Furthermore, the optimal usage for each ligand and associated nanoparticle will also be examined. This review will go into detail about pure noble metal nanoparticle, glucose, PEG, CTAB, Transferrin, Anti-Microbial Peptide, and Chitosan as coatings. All of these are commonly used among researchers. The absorptivity into various cell types in the brain along with future implications will be examined.

\section{Introduction}

Noble metal nanoparticles (NMNPs) including gold, silver, and platinum-based platforms are a revolutionary technology because of their size, shape, optoelectronic, and surface charge capabilities (Klębowski et al., 2018). The variability of these characteristics allows NMNPs to have various applications in drug delivery, imaging, and targeting (Azharuddin et al., 2019). Gold nanoparticles specifically have intrinsic qualities that cause them to be highly biocompatible (Bhattacharya \& Mukherjee, 2008). As a soft acid, gold binds strongly with bases like thiols and amine groups (Bhattacharya \& Mukherjee, 2008). The biocompatability of gold makes it an exceptional drug carrier. The most challenging aspects is passing the regulators of the brain: blood brain barrier (BBB) and the blood-cebrospinal fluid barrier, both of which regulates the ability of particles to enter the brain (Curtis et al., 2017). The BBB is formed by microvascular endothelium, pericytes and astrocytes, preventing the movement of larger hydrophilic molecules. $50 \mathrm{~nm}$ is a generally recognized upper limit on nanoparticle size for crossing the BBB (Gromnicova et al., 2013). However, nanoparticles are typically seen as anywhere from 1- $100 \mathrm{~nm}$. Nanoparticles can cross the BBB via multiple mechanisms including passive diffusion and endocytosis (Tripathi et al., 2015). Because the BBB is such an effective barrier, researchers and physicians turn to alternative more invasive methods to deliver drugs to the brain. Invasive methods include intercerebral or intracerebroventricular injections, which can be difficult on patients because of the risky nature of this procedure(Fullstone et al., 2016). NMNPs provide a safe way to cross the blood brain barrier, through passive diffusion or transcytosis, and target cells and protiens.

Ligands can act as cell entrance facilitator and majority of the time does not alter the biodistribution of the nanoparticles (Wiley et al., 2013). Nanoparticles are able to connect with multiple ligands on a single nanoparticle (Fullstone et al., 2016). By controlling nanoparticle ligand density and receptor ligand affinity, the binding of 
particular scenarios can be regulated allowing researchers to have control over aspects of the nanoparticle. For example if the ligand is of low affinity, then the nanoparticle will only stably bind if there is a high ligand density and high receptor availability (Fullstone et al., 2016). Furthermore, the number of ligands can also play a role in the nanoparticle's ability. Individual ligands reduce the affinity of the ligand to the receptor. Multiple targeting ligands can bind and the avidity and selectivity are increased (Wiley et al., 2013). These benefits allow researchers to prevent bonding in the blood stream and also account for other obstacles they may encounter.

We have performed a systematic review of the use of noble metal nanoparticles in the treatment of brain diseases. We limited our search specifically to gold, silver, and platinum nanoparticle formulations used to treat brainrelated diseases and injuries. We constructed a search query in PubMed taking advantage of Medical Subject Headings $(\mathrm{MeSH})$ terms to guide our search. Our final search query was:

((Metal Nanoparticles / therapeutic use) OR (Metal Nanoparticles / chemistry*) OR (Platinum / chemistry) OR (Silver / chemistry) OR (Gold / chemistry)) AND ((Astrocytes / drug effects) OR (Dendritic Spines / drug effects) OR (Hippocampus / drug effects*) OR (Neuroglia / drug effects*) OR (Neurons / drug effects*) OR (Blood-Brain Barrier / metabolism*) OR (Brain / metabolism*))

We have organized our literature search by the ligand type associated with the nanoparticle platforms. Ligands often have a strong impact on nanoparticle fate thus making them a natural method of categorization. We will examine NMNPs without ligands, conjugated with simple ligands, and conjugated with complex ligands. For the purposes of this review, we provide the following definitions for non-coated NMNPs, simple ligands, and complex ligands:

- Non-coated NMNPs: noble metal nanoparticles with no ligands physically or chemically bound to the surface.

- Simple ligands: small molecules $(<200 \mathrm{kDa})$ such as simple sugars.

- Complex ligands: biologically derived molecules such as proteins and DNA. All ligands being considered are found in figure 1.

\section{Non-coated Noble Metal Nanoparticles}

NMNPs have surface charges which affect how ligands are able to bond to them. However, the varying surface charge can also be both helpful and harmful when dealing with transportation to the brain. Coatings are used to prevent coagulation of particles in the blood stream which would prevent the particles from passing through the BBB. On the other hand, by changing the charge scientists predicted it would denature proteins (Bhattacharya \& Mukherjee, 2008). Non-coated NMNPs form a monolayer protected cluster, to denature proteins using their positive or negative charge. (Bhattacharya \& Mukherjee, 2008). Withought a ligand, if the NMNP were able to pass through the BBB it could be used to denature proteins. In contrast, the charge of a particle can also be harmful to the nanoparticles' capabilities. Positive and negative charges can also interact with proteins which create unwanted immune responses and more rapid blood clearance via increased uptake. By regulating the charge on a pure noble metal nanoparticle, NMNPs have a higher chance of passing through the BBB (Ji et al., 2019). Through ligands, the charge on NMNP's can be regulated and able to pass through the BBB. Pure NMNP's are able to use their charge to denature proteins which can be beneficial if that is the aim of an experimenter.

\section{Simple Ligands}

Throughout the narrative of simple ligands versus complex, I have defined simple ligands as polymers, which are short in length and simple sugars. Complex polymers are those with longer chains which make for longer ligands. Depending on the goal of the researchers and the dieases they are researching each ligand has its respective postives and negatives. A simple ligand, is more cost effective than a complex ligand. They also prevent the interaction between pure NP's and proteins in the blood stream which can hinder the nanoparticle from reaching the brain through interactions with white blood cells. 


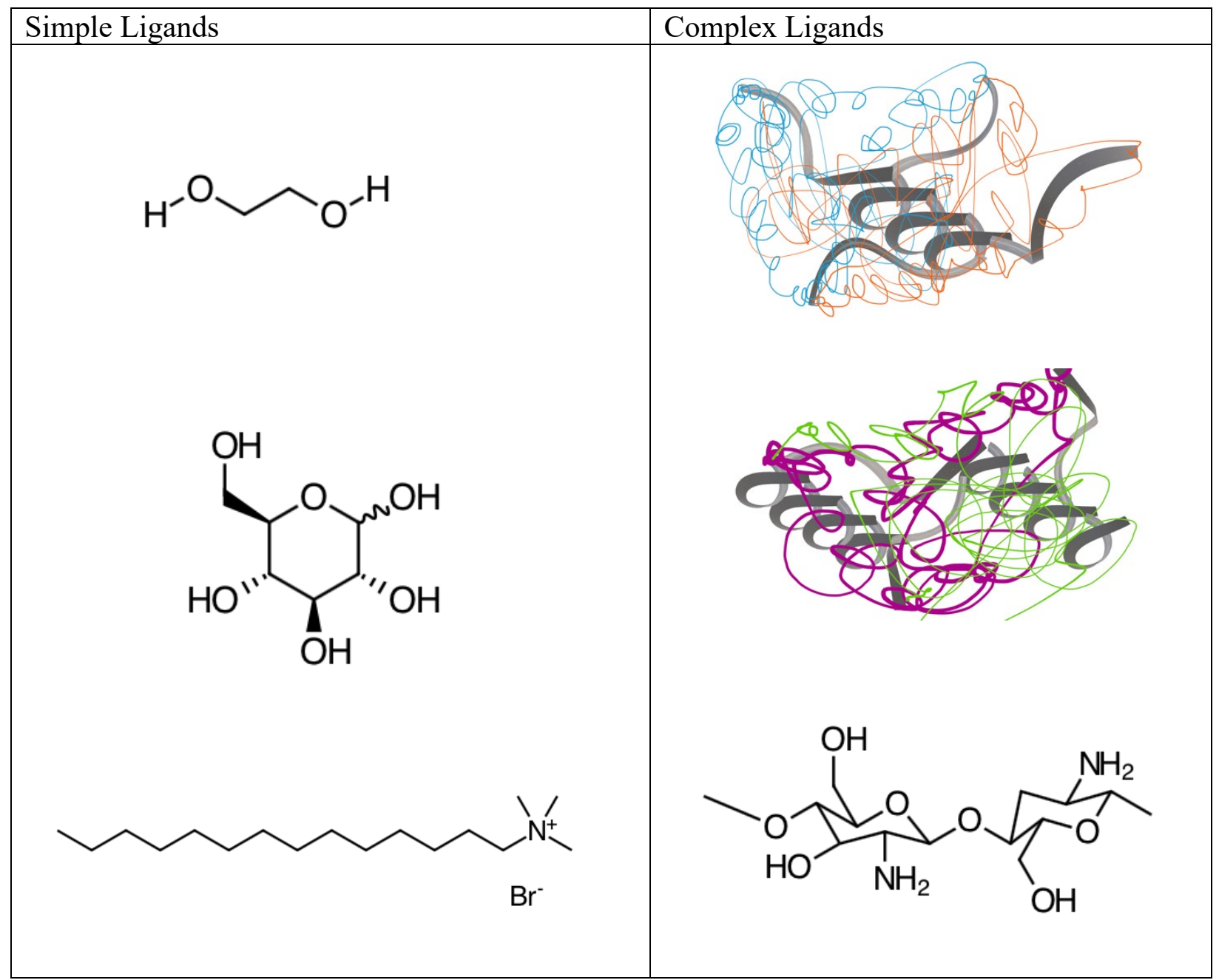

Figure 1. Different types of ligands commonly used in conjunction with noble metal nanoparticles. Starting at the top left and moving downward: (a). Polyethylene Glycol (b). Glucose (c). Cetyltrimethylammonium Bromide (d). Transferrin (e). Antimicrobial Peptides (f). Chitosan

\section{Glucose Coated Nanoparticles}

Glut-1 is found on the brain endothelium and in astrocytes. This led researchers to coat NMNPs in glucose so the nanoparticles are able to cross the endothelium, interact with astrocytes, and deliver drugs to the brain (Gromnicova et al., 2013). It was found that glyconanoparticles were able to prevent cell adhesion between the cells and pathogens. Gromnicova et al. also treated nanoparticles in endocytosis inhibitors to examine alternate transport mechanisms in the brain. They found that none of the treatments reduced the rate of nanoparticle transfer. The particles were transported through passive diffusion which was shown by the researchers coating nanoparticles in different polymers which would prevent endocytosis and seeing the rate of diffusion unchanged (Gromnicova et al., 2013). Contrary to their initial hypothesis, the glucose molecule was able to enter into cells through passive diffusion, exhibiting the ability of glucose as a ligand. (Gromnicova et al., 2013). This shows how ligands are able to determine which way a particle will pass through the BBB. In the case of glucose, nanoparticles coated in glucose take on the passive diffusion properties of glucose. 
The path of glucose coated nanoparticles was emphasized when glucose coated nanoparticles were injected in the left carotid artery. It was found that they circulated through the brain while keeping the BBB undisturbed. Nanoparticles were noticed in the contralateral cortex and the cytosol of endothelial cells. They were also found in the nucleus and the cytosol of glia cells and in neurons (Gromnicova et al., 2016). As a ligand, glucose was able to interact with neural cells and circumvent the BBB, as well as induce uptake in multiple cell types in the brain. Because they were present in each of these cell types it allows the possibility for nanoparticles to be further enhanced as targeted drug delivery systems specifically for neurodegenerative diseases (Gromnicova et al., 2016).

\section{Polyethylene Glycol (PEG)}

Polyethylene Glycol (PEG) is one of the most common coatings because of its affordability and its efficiency (D'souza \& Shegokar, 2016). PEG is an agent used to modify the surface of NMNPs to provide anchor points for biomolecule conjugation ( $\mathrm{Ji}$ et al., 2019). Furthermore, the PEGylation strategy is extensively used for increasing the protein lifespan in the bloodstream as well as for stabilizing protein structures (Huang et al., 2014). It prevents other nanoparticles or cells from accumulating and white cells from attacking the nanoparticles and filtering them out of the body. PEG can coat all different shapes, but it works particularly well with gold because it provides stability (Ji et al., 2019). Ji et al., coated gold nanospheres, nanorods and flowers with PEG. They examined the effect of PEG-coated nanoparticles on dendritic spine morphology in organotypic hippocampal slice cultures. It was found that the gold nanoparticle flowers coated in PEG are internalized by neurons as well as glial cells (Ji et al., 2019). In the study, they tested PEG and $\mathrm{CTAB}$ and found the optimal coating, size, and shape to effect spine density, which was AuNP-flower- CTAB and AuNP-rod-CTAB. Hutter et al. began to examine how microglia uptake gold nanoparticles. The found that microglial uptake of nanoparticles was surface coating-independent. Shape plays a larger role in the extent of uptake compared to surface coating (Hutter et al., 2010). For instance, PEG-coated nanoparticles are just as effective as CTAB-coated nanoparticles, but they can be tuned to increase internalization rates. Intra-nasal administration of PEGcoated gold nanorods significantly induced TLR2, which is associated with immunity and activation in microglia in vivo (Ji et al., 2019). A future application is this can be used for people with low immunities the nanoparticles could help boost immunity.

An potential issue with PEG is its cytotoxicity in excess. Hutter et al. predicted that shape would positively or negatively effect the percentage of cytotoxicity. Ji et al, found that Gold nanoparticles-flowers-PEG exerts greater toxicity in astrocytes compared to neurons and microglia. It was also found that spherical GNP's coated in both PEG and CTAB can be highly toxic and cause cell death. However, it was found that microglia were less sensitive to PEGconjugated gold nano-urchins and spheres in vitro compared to in vivo. This evidence further suggests that the toxic effects of gold nanoparticles is cell-type specific and must be evaluated in different cell types (Hutter et al., 2010). Another common issue with PEG is the lack of cell uptake (Hutter et al., 2010). However, PEG can be used in conjunction with other ligands to cross the BBB at higher rates. It is commonly used in conjunction with antimicrobial peptides and transferrin (D’souza \& Shegokar, 2016) (Sharma et al., 2016).

\section{Cetyltrimethylammonium bromide (CTAB)}

CTAB is a commonly used ligand because it has been shown to have a decrease in dendritic spines. The CTAB gold nanoparticles are able to alter lysosomal functions which have been closely related to dendritic spines (Maysinger \& Ji, 2019). It was found that AuNP flower- CTAB and AU np Rods CTAB decrease spine density in a concentration and time-dependent manner, and AU NP flowers CTAB can stimulate lysosome biogenesis. (Ji et al., 2019). Platinum nanoparticle aggregation was reduced when CTAB and NP9 was used as surfactants (Rai et al., 2016). CTAB is highly toxic in comparison to PEG. CTAB and CTAB-coated nanoparticles can lead to damage of cell membranes and mitochondria leading to cell death (Ji et al., 2019). 


\section{Complex Ligands}

\section{Transferrin}

Transferrin is a type of glycoprotein which allows for the transport of iron through the BBB (Fullstone et al., 2016). Transferrin grants NMNPs access to transferrin receptors initiating endocytosis. Transferrin density on the nanoparticle surface effects the ability of the nanoparticle to transport through the BBB (Fullstone et al., 2016). High nanoparticle transferrin density results in high affinity to brain endothelial cells, whereas low transferrin densities increase interactions with receptors on the BBB. If the avidity of the nanoparticles is too low, they do not bind to the transferrin receptor. But if the avidity is too high they are less available for release into the brain parenchyma (Wiley et al., 2013). By changing the avidity and concentration of transferrin, researchers are able to induce endocytosis in the most effective way possible to reach the brain.

Transferrin can be coupled with PEG to allow the nanoparticle to increase retention rates in the brain. The PEG coating prevents uptake by white blood cells, and transferrin mediates transportation through the BBB. PEG and transferrin are commonly together due to the ability of PEG to bind to lysine residues on transferrin (Bellocq et al., 2003). This combination of simple and complex ligands better facilitates the transport across the BBB. Ligand density and avidity can be used to modulate transport across the BBB.

A number of factors must be taken into account during ligand selection, including receptor expression, competition with endogenous ligands, efficacy of ligand-receptor interactions, and receptor half-life or rate of recycling to the plasma membrane after endocytosis. For the transferrin receptor there are a number of other synthesized ligands which can be used such as Ox26 antibody, R17217 antibody, and 8D3 antibody. Endogenous ligands only bond to one receptor, whereas synthesized ligands can bind to over 30 different proteins (Fullstone et al., 2016). These promiscuous ligands can bond to unintended substrates. However, endogenous ligands must take into account shape and size in order to bond the endogenous ligands to the nanoparticle properly (Fullstone et al., 2016). Without ligands, the nanoparticles will not be able to transfuse through the blood brain barrier and will coagulate or be attacked by white blood cells.

Gold nanoparticles have the capability to complex a drug payload and transferrin and monoclonal antibody ligands to maximize nanoparticle effectiveness. Transferrin causes the nanoparticles to undergo endocytosis. The monoclonal antibody allows the for the nanoparticle to target certain cell types. And the drug conjugate allows researchers to tune the nanoparticle platform for specific diseases (Tripathi et al., 2015). This has future applications to be used in cancer because it will directly be able to find certain cells and cause cell death without harming the entire body like many current cancer therapies.

\section{Antimicrobial-Peptide-Conjugated}

Antimicrobial peptides (AMPs) are a potential solution to the growing issue of drug resistance (Rajchakit et al., 2017). AMPs are used to kill bacteria and fungi (Rajchakit et al., 2017). Gold nanoparticles are especially compatible with AMPs because they have a simple synthesis process, easy functionalization, capability of crossing cell membranes, increased drug release at the site of infection due to high surface to volume, ability to kill the pathogens by inhibiting multiple bacteriological targets. Drug-conjugated gold nanoparticles have a stronger antibacterial activity in comparison to a naked gold nanoparticles (Rajchakit et al., 2017). Gold nanoparticles can non-covalently bind to antibiotics through a single-step conjugation. The presence of free amino groups show a high affinity towards the surface of gold and causes gold nanoparticle aggregation (Rajchakit et al., 2017). The simple synthesis process makes them readily usable and can be made at scale. Silver nanoparticles are commonly known to have some antibacterial properties. They have been used in conjunction with different substances such as poly(ethyleneimine), amoxicillin, polysaccharides, peptides, surfactants, and polymers (Kumar et al., 2012). When the silver nanoparticles were 
conjugated to antimicrobial peptides, the coated nanoparticles were able to increase the antibacterial activity against bacteria providing an alternative therapy for topical infections (Morales-Avila et al., 2017). Both silver and gold can be used in conjunction with AMPs.

Gold nanoparticles can disrupt membrane potential and disrupt ATPase inhibition which would lead to the reduction in the level of ATP(Rizvi et al., 2018). In the second mechanism, the ribosomal subunit bonded to the tRNA which inhibited production and caused a disruption of the transcription mechanism (Rizvi et al., 2018). The ability of gold nanoparticles to disrupt the transcription mechanism prevents the continued creation of proteins associated with certain diseases. When couple with AMP, this capability could target bacterial proteins. AMPs were found to be compatible with PEG. The ligand PEG stabilizes the secondary structure of a helical peptide, a 33-residue designed coiled peptide, in which polymers with higher molecular weight increase the peptide helicity (Jafari et al., 2019) . Helicity was proved to play a crucial role on peptide specificity and biological activities. (Huang et al., 2014). Increasing helicity by pairing with PEG allows AMP to increase it capabilities. Pairing AMP with PEG minimizes the effects of AMPs on healthy cells (Jafari et al., 2019). However, AMPs have some drawbacks. They have low enzymic stability and permeability across biological barriers (Rajchakit et al., 2017). However, when antimicrobial peptides were combined with transferrin or another biological compatible protein, AMPs could pass through the BBB.

\section{Chitosan}

Chitosan has low toxicity, high biocompatibility, biodegradability, antimicrobial activity, and low immunogenicity (Mili et al., 2018). Chitosan is a positively charged molecule with amino groups, and as such, it can bind to molecules with negative charges (Razavi et al., 2019). Chitosan is able to bind with nanoparticles through ionic interactions. It is commonly used in conjunction with nerve growth factor (NGF) to help regenerate tissues using stem cells. NGF by itself lacks the ability to penetrate cells. However, when coupled with a nanoparticle carrier, it can regenerate cells (Mili et al., 2018). Chitosan can be used for neurological diseases to heal and regenerate cells. It was found to regenerate canine cells with future applications in humans. However the amount of necrotic cells did increase (Mili et al., 2018) showing that like many of the other coatings there are adverse reactions.

Chitosan has also been used in conjunction with titanium dioxide nanoparticles (Huerta-García et al., 2015). When coated in chitosan, titanium dioxide particles are rendered non-toxic. The nanoparticles' small size facilitates uptake into cells and transcytosis across epithelial and endothelial cells into the blood and lymphatic circulation. Chitosan-coated titanium dioxide nanoparticles were internalized by coating them in Cyt-D, to prevent endocytosis, showing that the nanoparticles were able to move through alternative methods. It lowered nanoparticle internalization by 60 and 65 percent in U373 and C6 cell lines, respectively. Chitosan also lowered the uptake in glial cells (Hsiao et al., 2016). Chitosan-coated NMNPs can release a Cyt-D substance, a cytochalasin, into harmful proteins so they cannot be up taken by glial cells. This can prevent harmful diseases from infecting the brain and affecting it as heavily as it does.

\section{Future Directions}

Their minimally invasive nature and compatibility allows the nanoparticles to be modified to specific diseases. NMNPs are commonly seen as the future of cancer research specifically brain tumors. Because brain tumors are so invasive, they grow rapidly (Tripathi et al., 2015). The functionalized nanoparticles which will allow the desired molecule to bind on to it and the antibody to bind on to that. This will allow the nanoparticle to release the drug directly attacking the tumor (Tripathi et al., 2015). However, a massive issue facing many studies is the lack of research making it to the clinical level. Not many researchers progress their research past the in vitro and in vivo stages. The use of NMNP's will be revolutionary for cancer research and allow for drugs to kill tumors without attacking every cell in the body. Furthermore, there is still a need to research into the biodegradability, porosity, and 
cytotoxicity. Many researchers are continuing to optimize their therapeutic combinations for creating NMNP's and the use of proteins as stabilizers, such as Chitosan, is continued to be examined (Kong et al., 2017).

In the past years, newer methods of creating gold and silver nanoparticles have become prevalent. Some researchers have used stirring chloroauric acid solutions with reducing agents which resulted in $\mathrm{Au}^{3+}$ ions becoming neutral ions (Swain et al., 2016). In the creation of silver nanoparticles, the chemical synthesis has been shown to have short comings and has resulted in a biological method being tested(Xu et al., 2020). This methodology requires the use of bacteria, fungi, and algae but has been determined to be more environmentally friendly (Xu et al., 2020). In all of the NMNP's, the long-term effects of the particles are continued to be examined because they are unknown. The trend in the nanoscience field is to examine the effect on in vivo cells and continue to develop NMNP's so they can be used in drug delivery. Without mitigating the cytotoxicity effects and continuing research into the clinical phases, NMNP's could not be used as a drug delivery system

\section{Conclusion}

NMNPs can be used as effective drug delivery platforms to the brain. Ligands can change the behavior of NMNPs, including biodistribution, protein adhesion, diffusive ability, and cell internalization. Using NMNP's is especially beneficial when needing to pass through biological barriers. Furthermore, it allows for absorption into specific cell types and in order to target cancer cells, NMNP's would allow for the targeting and drug distribution to occur. Choosing the proper ligand is dependent upon the goals of the researcher and the type of nanoparticles they are using. When dealing with different diseases it is important to choose the ligand which is best suited to fight that diseases. Ligands can change the capabilities of the nanoparticles and can also help prevent coagulation. However, they also have downsides such as the cytotoxic effect of coating such as PEG and CTAB which can cause mass cell death if used in excess. In order to mitigate, these effects experimenters recommend using a smart drug delivery approach or also looking at multiple forms of the coating. Specifically, with PEG, there is an issue with resistance to the coating but through mPEG or hydroxyl- PEG these effects will be mitigated. CTAB's toxicity was able to be mitigated through adding a coating of PEG. Glucose and Chitosan was not found to have cytotoxic effect but more research is needed to examine if they can fully mitigate the cytotoxicity in the NMNP's. Glucose and transferrin are extremely effective in crossing the BBB and gaining entrance into cell types through endocytosis and passive diffusion. NMNPs have the capabilities to be used in the brain and will revolution the field of drug delivery to the brain. I believe the most impactful and economically sound ligand would be PEG. Due to the fact CTAB, has high cytotoxicity which can only be mitigated by PEG, I believe it would be more useful to use PEG. Although antimicrobial peptides, chitosan, transferrin, and glucose are all useful in their respective scenarios, there is more testing to be done especially in the limitations of each of the coatings.

\section{Acknowledgments}

I would like to acknowledge Dr. Chad Curtis and thank him for his guiding presence in the creation of this paper. Without his mentorship, this paper would not have been possible. His ability to explain complicated notions has allowed me a deeper understanding into the world of biochemistry.

\section{References}

_A repertoire of biomedical applications of noble metal nanoparticles Mohammad Azharuddin, Geyunjian H. Zhu, Debapratim Das, Erdogan Ozgurd, Lokman Uzund, Anthony P.F. Turner and Hirak K Patra 
Recent Developments in Antimicrobial-Peptide-Conjugated Gold Nanoparticles.pdf. (n.d.).

Azharuddin, M., Zhu, G. H., Das, D., Ozgur, E., Uzun, L., Turner, A. P. F., \& Patra, H. K. (2019). A repertoire of biomedical applications of noble metal nanoparticles. Chemical Communications, 55(49), 6964-6996. https://doi.org/10.1039/c9cc01741k

Bellocq, N. C., Pun, S. H., Jensen, G. S., \& Davis, M. E. (2003). Transferrin-Containing, Cyclodextrin PolymerBased Particles for Tumor-Targeted Gene Delivery. Bioconjugate Chemistry, 14(6), 1122-1132. https://doi.org/10.1021/bc034125f

Bhattacharya, R., \& Mukherjee, P. (2008). Biological properties of "naked" metal nanoparticles. Advanced Drug Delivery Reviews, 60(11), 1289-1306. https://doi.org/10.1016/j.addr.2008.03.013

Curtis, C., Zhang, M., Liao, R., Wood, T., \& Nance, E. (2017). Systems-level thinking for nanoparticle-mediated therapeutic delivery to neurological diseases. Wiley Interdisciplinary Reviews: Nanomedicine and Nanobiotechnology, 9(2). https://doi.org/10.1002/wnan.1422

D’souza, A. A., \& Shegokar, R. (2016). Polyethylene glycol (PEG): a versatile polymer for pharmaceutical applications. Expert Opinion on Drug Delivery, 13(9), 1257-1275. https://doi.org/10.1080/17425247.2016.1182485 Fullstone, G., Nyberg, S., Tian, X., \& Battaglia, G. (2016). From the Blood to the Central Nervous System: A Nanoparticle's Journey Through the Blood-Brain Barrier by Transcytosis. In International Review of Neurobiology (1st ed., Vol. 130). Elsevier Inc. https://doi.org/10.1016/bs.irn.2016.06.001

Gromnicova, R., Davies, H. A., Sreekanthreddy, P., Romero, I. A., Lund, T., Roitt, I. M., Phillips, J. B., \& Male, D. K. (2013). Glucose-coated gold nanoparticles transfer across human brain endothelium and enter astrocytes in vitro. PLoS ONE, 8(12). https://doi.org/10.1371/journal.pone.0081043

Gromnicova, R., Yilmaz, C. U., Orhan, N., Kaya, M., Davies, H., Williams, P., Romero, I. A., Sharrack, B., \& Male, D. (2016). Localization and mobility of glucose-coated gold nanoparticles within the brain. Nanomedicine, 11(6), 617-625. https://doi.org/10.2217/nnm.15.215

Hsiao, I. L., Chang, C. C., Wu, C. Y., Hsieh, Y. K., Chuang, C. Y., Wang, C. F., \& Huang, Y. J. (2016). Indirect effects of TiO2 nanoparticle on neuron-glial cell interactions. Chemico-Biological Interactions, 254, 34-44. https://doi.org/10.1016/j.cbi.2016.05.024

Huang, Y., He, L., Li, G., Zhai, N., Jiang, H., \& Chen, Y. (2014). Role of helicity of $\alpha$-helical antimicrobial peptides to improve specificity. Protein and Cell, 5(8), 631-642. https://doi.org/10.1007/s13238-014-0061-0 Huerta-García, E., Márquez-Ramírez, S. G., Ramos-Godinez, M. del P., López-Saavedra, A., Herrera, L. A., Parra, A., Alfaro-Moreno, E., Gómez, E. O., \& López-Marure, R. (2015). Internalization of titanium dioxide nanoparticles by glial cells is given at short times and is mainly mediated by actin reorganization-dependent endocytosis. NeuroToxicology, 51, 27-37. https://doi.org/10.1016/j.neuro.2015.08.013

Hutter, E., Boridy, S., Labrecque, S., Lalancette-Hébert, M., Kriz, J., Winnik, F. M., \& Maysinger, D. (2010). Microglial response to gold nanoparticles. ACS Nano, 4(5), 2595-2606. https://doi.org/10.1021/nn901869f

Jafari, M., Doustdar, F., \& Mehrnejad, F. (2019). Molecular Self-Assembly Strategy for Encapsulation of an Amphipathic $\alpha$-Helical Antimicrobial Peptide into the Different Polymeric and Copolymeric Nanoparticles. Journal of Chemical Information and Modeling, 59(1), 550-563. https://doi.org/10.1021/acs.jcim.8b00641 
Ji, J., Moquin, A., Bertorelle, F., KY Chang, P., Antoine, R., Luo, J., McKinney, R. A., \& Maysinger, D. (2019). Organotypic and primary neural cultures as models to assess effects of different gold nanostructures on glia and neurons. Nanotoxicology, 13(3), 285-304. https://doi.org/10.1080/17435390.2018.1543468

Klębowski, B., Depciuch, J., Parlińska-Wojtan, M., \& Baran, J. (2018). Applications of noble metal-based nanoparticles in medicine. International Journal of Molecular Sciences, 19(12). https://doi.org/10.3390/ijms19124031

Kong, F. Y., Zhang, J. W., Li, R. F., Wang, Z. X., Wang, W. J., \& Wang, W. (2017). Unique roles of gold nanoparticles in drug delivery, targeting and imaging applications. Molecules, 22(9). https://doi.org/10.3390/molecules22091445

Kumar, C. G., Mamidyala, S. K., Reddy, M. N., \& Reddy, B. V. S. (2012). Silver glyconanoparticles functionalized with sugars of sweet sorghum syrup as an antimicrobial agent. Process Biochemistry, 47(10), 1488-1495. https://doi.org/10.1016/j.procbio.2012.05.023

Maysinger, D., \& Ji, J. (2019). Nanostructured Modulators of Neuroglia. Current Pharmaceutical Design, 25(37), 3905-3916. https://doi.org/10.2174/1381612825666190912163339

Mili, B., Das, K., Kumar, A., Saxena, A. C., Singh, P., Ghosh, S., \& Bag, S. (2018). Preparation of NGF encapsulated chitosan nanoparticles and its evaluation on neuronal differentiation potentiality of canine mesenchymal stem cells. Journal of Materials Science: Materials in Medicine, 29(1), 1-13. https://doi.org/10.1007/s10856-017-6008-2

Morales-Avila, E., Ferro-Flores, G., Ocampo-Garciá, B. E., López-Téllez, G., López-Ortega, J., Rogel-Ayala, Di. G., \& Sánchez-Padilla, Di. (2017). Antibacterial Efficacy of Gold and Silver Nanoparticles Functionalized with the Ubiquicidin (29-41) Antimicrobial Peptide. Journal of Nanomaterials, 2017. https://doi.org/10.1155/2017/5831959

Rai, M., Ingle, A. P., Birla, S., Yadav, A., \& Santos, C. A. Dos. (2016). Strategic role of selected noble metal nanoparticles in medicine. Critical Reviews in Microbiology, 42(5), 696-719. https://doi.org/10.3109/1040841X.2015.1018131

Razavi, S., Seyedebrahimi, R., \& Jahromi, M. (2019). Biodelivery of nerve growth factor and gold nanoparticles encapsulated in chitosan nanoparticles for schwann-like cells differentiation of human adipose-derived stem cells. Biochemical and Biophysical Research Communications, 513(3), 681-687. https://doi.org/10.1016/j.bbrc.2019.03.189

Rizvi, S. M. D., Hussain, T., Ahmed, A. B. F., Alshammari, T. M., Moin, A., Ahmed, M. Q., Barreto, G. E., Kamal, M. A., \& Ashraf, G. M. (2018). Gold nanoparticles: A plausible tool to combat neurological bacterial infections in humans. Biomedicine and Pharmacotherapy, 107(April), 7-18. https://doi.org/10.1016/j.biopha.2018.07.130 Sharma, G., Lakkadwala, S., Modgil, A., \& Singh, J. (2016). The role of cell-penetrating peptide and transferrin on enhanced delivery of drug to brain. International Journal of Molecular Sciences, 17(6). https://doi.org/10.3390/ijms17060806

Swain, S., Sahu, P., Beg, S., \& Babu, S. (2016). Nanoparticles for Cancer Targeting: Current and Future Directions. Current Drug Delivery, 13(8), 1290-1302. https://doi.org/10.2174/1567201813666160713121122 
Tripathi, R. M., Shrivastav, A., \& Shrivastav, B. R. (2015). Biogenic gold nanoparticles: As a potential candidate for brain tumor directed drug delivery. Artificial Cells, Nanomedicine and Biotechnology, 43(5), 311-317. https://doi.org/10.3109/21691401.2014.885445

Wiley, D. T., Webster, P., Gale, A., \& Davis, M. E. (2013). Transcytosis and brain uptake of transferrin-containing nanoparticles by tuning avidity to transferrin receptor. Proceedings of the National Academy of Sciences of the United States of America, 110(21), 8662-8667. https://doi.org/10.1073/pnas.1307152110

Xu, L., Wang, Y. Y., Huang, J., Chen, C. Y., Wang, Z. X., \& Xie, H. (2020). Silver nanoparticles: Synthesis, medical applications and biosafety. Theranostics, 10(20), 8996-9031. https://doi.org/10.7150/thno.45413 